\title{
The CUORE and CUORE-0 experiments at LNGS
}

\author{
Claudia Tomei* \\ for the CUORE Collaboration \\ INFN - Sezione di Roma, Roma I-00185, Italy \\ E-mail: claudia.tomei@romal.infn.it
}

The Cryogenic Underground Observatory for Rare Events (CUORE) is a large-scale double beta decay experiment based on cryogenic bolometers currently taking data at the Gran Sasso National Laboratory (LNGS). Its primary goal is to observe the neutrinoless double beta decay of ${ }^{130} \mathrm{Te}$ and measure the Majorana neutrino mass with a projected sensitivity reaching the so-called inverted mass hierarchy region. Here we discuss the CUORE challenges and the successful installation and commissioning of the detector, as well as the physics potential of CUORE. We also review the physics results of the CUORE prototype CUORE- 0 .

The European Physical Society Conference on High Energy Physics

5-12 July, 2017

Venice

${ }^{*}$ Speaker. 


\section{Introduction}

One of the most sensitive probes for physics beyond the Standard Mode is the neutrinoless double-beta $(0 v \beta \beta)$ decay, a lepton-number-violating process hypothesized for the first time by Pontecorvo in 1967 [1]. The observation of $0 v \beta \beta$ decay would demonstrate that lepton number is not conserved, establish neutrinos as Majorana particles and set constraints on the effective neutrino Majorana mass, while providing information on the absolute neutrino mass scale. Many experiments worldwide are searching for this decay [2, 3, 4, 5] but no evidence has decisively been found.

One general experimental approach for detecting $0 v \beta \beta$ decay is to measure the sum energy of the two electrons emitted in the final state. In this case, the signature is a peak at Q-value. The decay rate, assuming the simplest mechanism of a massive Majorana neutrino exchange, is proportional to the square of the so called effective Majorana mass $\left|m_{e e}\right|$ :

$$
\frac{1}{T_{1 / 2}^{0 v}}=\frac{\left|m_{e e}\right|^{2}}{m_{e}^{2}} G^{0 v}\left|M^{0 v}\right|^{2}
$$

where $\mathrm{T}_{1 / 2}$ is the decay half-life, $m_{e}$ is the electron mass, $G^{0 v}$ is the two-body phase-space factor, and $M^{0 v}$ is the $0 v \beta \beta$ nuclear matrix element (NME). While $G^{0 v}$ can be calculated with reasonable accuracy [7], the NME value strongly depends on the nuclear model $[8,9,10,11,12]$ and the current calculations are still affected by considerable uncertainty in the calculation of $\left|m_{e e}\right|$ from experimental measurements or limits on the half-life.

Among the many experimental techniques applied to the $0 v \beta \beta$ searches, cryogenic bolometers (ultra-cold crystals functioning as highly sensitive calorimeters) have numerous advantages. They provide excellent efficiency (being solid state detectors the two electrons are mostly contained in the bulk), excellent energy resolution, and they offer a wide choice of detector material. The working principle is the following: the energy deposited by a particle interaction in the crystal is converted to a measurable temperature variation. This temperature rise is measured by a thermometer (semiconductor thermistor) glued onto the surface of the absorber.

CUORE [13, 14] stands for Cryogenic Underground Observatory for Rare Events and exploits the bolometric technique $[15,16]$ in an array of $9885 \times 5 \times 5 \mathrm{~cm}^{3}$ nat $\mathrm{TeO}_{2}$ crystals to search for the $0 v \beta \beta$ decay of ${ }^{130} \mathrm{Te}$. The experiment is installed at Laboratori Nazionali del Gran Sasso (LNGS) of INFN and represents the largest array of cryogenic bolometers ever built, with a total mass of $742 \mathrm{~kg}$ (206 kg of the emitting double-beta isotope ${ }^{130} \mathrm{Te}$ ).

CUORE was preceded by two prototypes: Cuoricino [17, 18], that operated from 2003 to 2008 and set a limit on the half-life of $0 v \beta \beta$ of ${ }^{130} \mathrm{Te}$ at $T_{1 / 2}^{0 v}>2.8 \cdot 10^{24}$ y @ 90\% C.L. [19], and CUORE-0 [17, 18], that operated from 2013 to 2015 and with only half of the Cuoricino exposure was able to set a comparable limit thanks to the lower background. The two experiments yielded a combined result of $T_{1 / 2}^{0 v}>4.0 \times 10^{24} \mathrm{yr}$ at $90 \%$ C.L. limit, which is the most stringent limit to date on the ${ }^{130} \mathrm{Te} 0 v \beta \beta$ half-life.

\section{The CUORE experiment}

CUORE is designed to search for the $0 v \beta \beta$ decay of ${ }^{130} \mathrm{Te}$. The choice of the absorber $\left(\mathrm{TeO}_{2}\right)$ 
is driven by the excellent bolometric properties of this material, the high natural abundance of the $\beta \beta$ emitting isotope ${ }^{130} \mathrm{Te}(\sim 34 \%$, the highest among the $\beta \beta$ emitting isotopes used to date) and the reasonably high $Q_{\beta}$, almost above the energy spectrum of natural radioactivity. CUORE scales up the sensitivity of Cuoricino and CUORE-0 by increasing the mass and at the same time improving the energy resolution and reducing the background, with the goal of achieving a count rate in the region of interest (ROI) of 0.01 counts $/(\mathrm{keV} \cdot \mathrm{kg} \cdot \mathrm{y}$ ) and a FWHM of $5 \mathrm{keV}$ at the Qvalue. The CUORE detector array is cooled down to $10 \mathrm{mK}$ in a cryogenic apparatus consisting of a custom made cryostat with six nested copper shields, a cryogen free cooling system with five pulse tubes, a powerful dilution refrigerator, and a fast cooling system for pre-cooling. The custom made CUORE cryostat represents a huge technological challenge accomplished by the CUORE collaboration: to operate a huge bolometric array, in an extremely low radioactivity and low vibrations environment. The low radioactivity level was kept under control using radio-pure materials and clean assembly procedures. An independent suspension of the detector array from the dilution unit guarantees a low vibrational noise.

Reducing the rate of spurious counts that can hinder the faint $0 v \beta \beta$ signal is another huge challenge for experiments such as CUORE. To this aim, only carefully selected radio-pure materials are used in the detector region, namely high-purity low-background copper and PTFE. The experience of Cuoricino and CUORE-0 has shown that an important fraction of the background in the ROI is due to degraded alpha from U/Th contaminations on copper and crystal surfaces. The CUORE crystals are produced from the Shanghai Institute of Ceramics, Chinese Academy of Sciences (SICCAS) following a strict radiopurity control protocol [20] to limit bulk and surface contaminations. They were transported at sea level to LNGS in vacuum bags and boxes and finally stored underground in nitrogen fluxed cabinets. A complex cleaning procedure developed at Legnaro National Laboratory of INFN was applied to copper components facing the detectors. This is defined TECM (Tumbling, Electropolishing, Chemical etching, and Magnetron plasma etching). The compact design and the granularity of the CUORE array provides an additional background reduction through self-shielding and anti-coincidence between the detectors.

To shield against the external radioactivity, the detector features a lead shield, divided into several layers, the innermost ones made from ancient low-radioactivity Roman lead. A neutron shield surrounds the whole cryostat.

The assembly of all the 19 CUORE towers was completed in 2014 inside the CUORE clean room in Hall A of LNGS. Every step of the assembly (gluing of the thermistors onto the crystals, wire bonding, cabling and mounting) was carried out inside a dedicated glove boxe flushed with nitrogen [22]. The cryogenic system commissioning was completed in March 2016. All the components were well thermalised at the different stages reaching a stable base temperature of $6.3 \mathrm{mK}$ on a month scale, with a cooling power of more than $3 \mu \mathrm{W}$ at $10 \mathrm{mK}$. During the first cool down, a full test of the readout chain was performed using a tower with 8 crystals (the so-called Minitower). The test demonstrated a good functioning of electronics, DAQ and detector calibration system. No unaccounted background sources were seen.

After the completion of the initial cool down, we installed and commissioned the lateral and bottom roman lead shielding. The installation of the towers began in August 2016 and lasted one month (see Fig. 1). It was performed in a radon-free clean room. From September to November 2016 the cryostat closure took place and the cooldown started on Dec. 5, 2016. CUORE data 


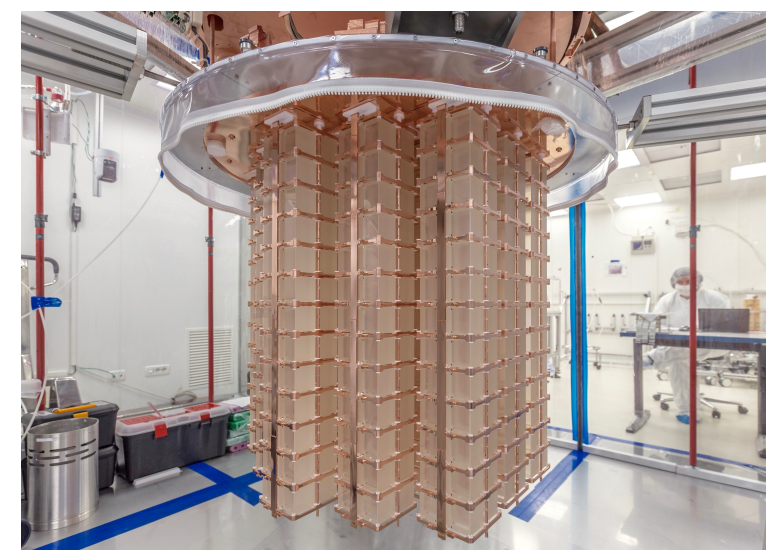

Figure 1: Completed installation of the CUORE towers.

taking finally began in April 2017.

The expected CUORE sensitivity at $90 \%$ C.L. is $T_{1 / 2}^{0 v}>2 \cdot 10^{25}$ after 3 months and $T_{1 / 2}^{0 v}>$ $9 \cdot 10^{25} \mathrm{y}$ in 5 years [26]. This corresponds to a limit on the Majorana neutrino mass in the range 50-130 meV, that approaches the so-called inverted mass hierarchy region.

\section{CUORE-0 results and projected background index for CUORE}

CUORE-0 was the first CUORE-like tower and CUORE technical prototype, assembled from detector components manufactured, cleaned and stored following CUORE protocols. Technical details and initial performances of CUORE-0 can be found in [23]. CUORE-0 data taking lasted from March 2013 to March 2015, for a total $\mathrm{TeO}_{2}$ statistics of $35.2 \mathrm{~kg} \cdot \mathrm{y}$ or $9.8 \mathrm{~kg} \cdot \mathrm{y}$ of ${ }^{130} \mathrm{Te}$. When combined with the $19.75 \mathrm{~kg} \cdot \mathrm{y}$ exposure of ${ }^{130} \mathrm{Te}$ from the Cuoricino experiment, CUORE-0 [24] set a Bayesian $90 \%$ C.L. limit on the ${ }^{130} \mathrm{Te} 0 v \beta \beta$ decay half-life of $T_{1 / 2}^{0 v}>4.0 \times 10^{24} \mathrm{yr}$. It also achieved a FWHM of $5.1 \pm 0.3 \mathrm{keV}$, which matches the CUORE FWHM goal.

A Montecarlo study of the CUORE-0 background spectrum was published in [27]. We successfully reconstructed the CUORE-0 background using 57 sources modeled with a detailed Geant4 based Monte Carlo simulation. The activities of the sources were determined by a Bayesian fit of the experimental spectra of different multiplicities with a linear combination of simulated source spectra. Fig. 2 shows the fit result compared with the CUORE-0 spectrum of single multiplicity. The background reconstruction allowed us to measure the ${ }^{130} \mathrm{Te} 2 v \beta \beta$ decay with high accuracy. From an exposure of $33.4 \mathrm{~kg} \cdot \mathrm{y}$ of $\mathrm{TeO}_{2}$, the half-life was determined to be $T_{1 / 2}^{2 v}=[8.2 \pm 0.2$ (stat.) \pm 0.6 (syst.) $] \times 10^{20} \mathrm{y}$.

To evaluate the expected background in CUORE, we performed a further detailed Monte Carlo simulation, based on results from environmental background measurements at LNGS, material screening for CUORE, and background sources from the CUORE-0 background model (see Fig. 3). The total projected background index in the ROI of CUORE is equal to $1.02 \pm 0.03$ (stat) ${ }_{-0.10}^{+0.23}$ (syst) $\times 10^{-2}$ counts $/ \mathrm{keV} / \mathrm{kg} /$ year. 


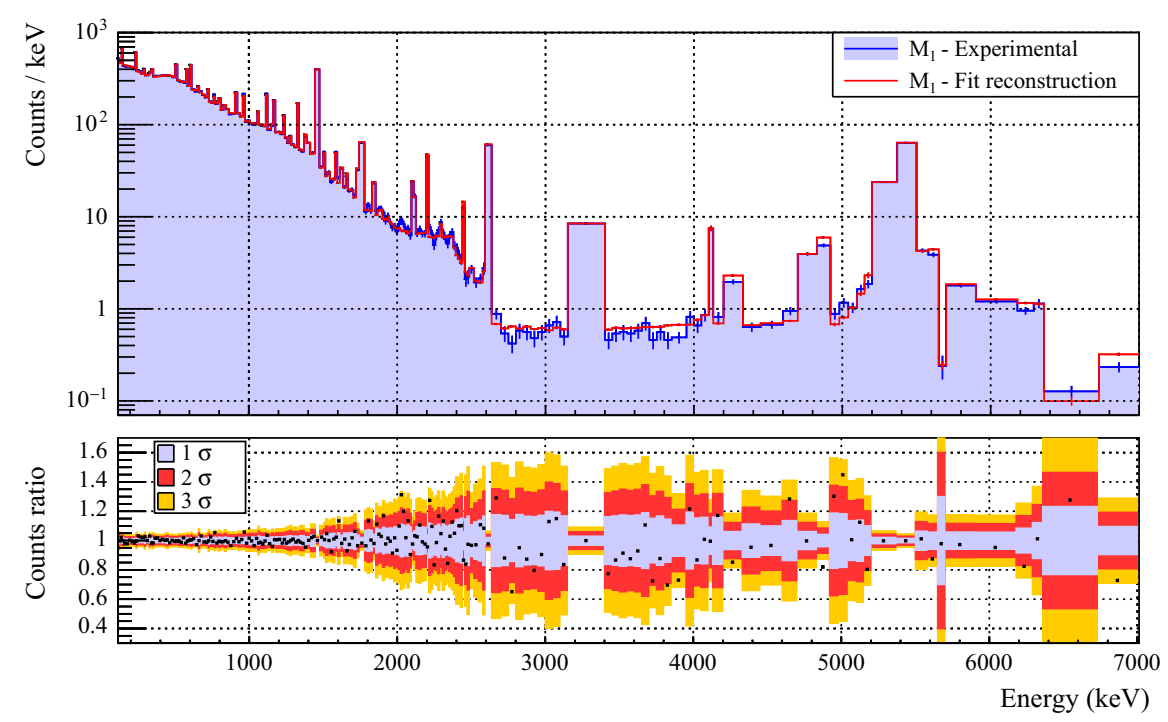

Figure 2: CUORE-0 background model fit result compared to the CUORE-0 spectrum of single multiplicity.
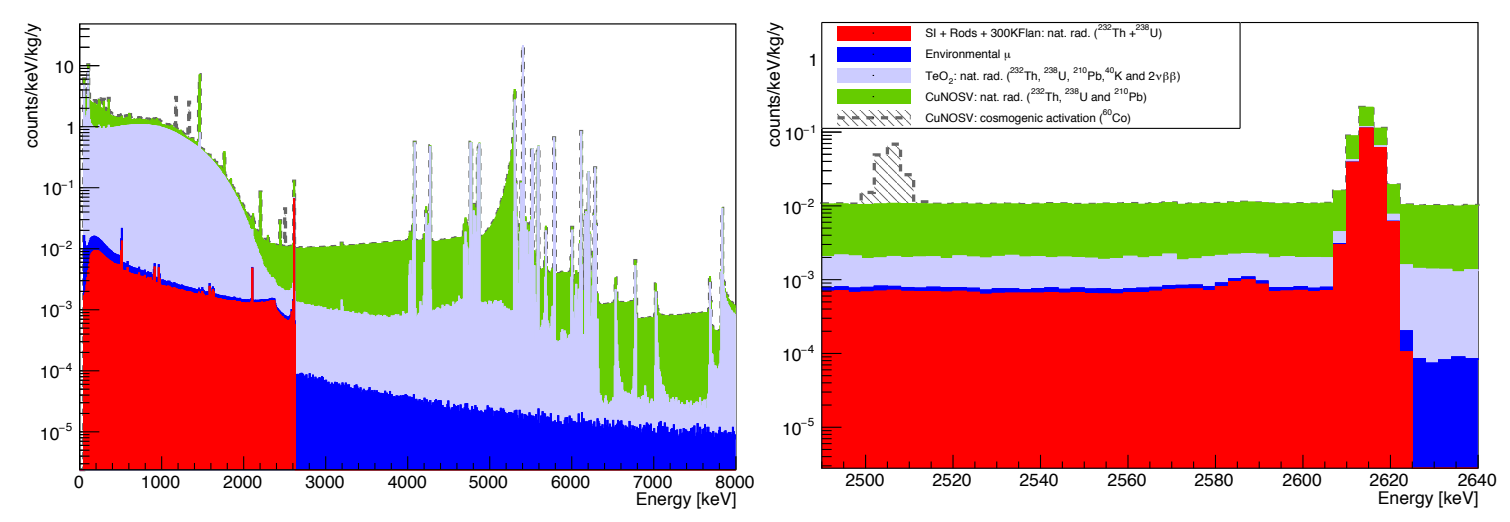

Figure 3: Projected background for the CUORE experiment, evaluated with a Monte Carlo simulation based on results from environmental background measurements at LNGS, material screening for CUORE, and background sources from the CUORE-0 background model.

\section{Conclusions}

$\mathrm{TeO}_{2}$ cryogenic bolometers offer a well-established, competitive technique in the search for neutrino-less double beta decay. The CUORE prototype CUORE-0 achieved its energy resolution and background level goals, surpassing the sensitivity of the former prototype, Cuoricino, in half the time. CUORE-0 also validated the CUORE assembly technology and background model and set the most stringent limit to date on the ${ }^{130} \mathrm{Te} 0 v \beta \beta$ decay half-life, as well as the most precise measurement of the $2 v \beta \beta$ decay half-life. CUORE is the first $0 v \beta \beta$ cryogenic experiment at the ton-scale. The detector installation and cool-down is completed and CUORE is now taking data. 


\section{References}

[1] Neutrino Experiments and the Problem of Conservation of Leptonic Charge, B. Pontecorvo, Sov. Phys. JETP 26, 984 (1968).

[2] Review of double beta experiments, X. Sarazin, arXiv:1210.7666v1 [physics.ins-det].

[3] Neutrinoless Double-Beta Decay, A. Giuliani, A. Poves, Advances in High Energy Physics 2012, 857016, (2012), http://dx.doi.org/10.1155/2012/857016.

[4] Challenges in Double Beta Decay, O. Cremonesi, M. Pavan, Advances in High Energy Physics, 2014, 951432, (2013), http://dx.doi.org/10.1155/2014/951432.

[5] Double beta decay experiments: current status and prospects, A. S. Barabash, Phys. Usp. 57, 482 (2014), https://doi.org/10.3367/UFNe.0184.201405f.0524.

[6] Neutrinoless double-beta decay: A probe of physics beyond the Standard Model, S.M. Bilenky, C. Giunti, Int. J. of Mod. Phys. A 30, 1530001 (2015), https://doi.org/10.1142/S0217751X1530001X.

[7] Phase-space factors for double-beta decay, J. Kotila and F. Iachello, Phys. Rev. C 85, 034316 (2012), https://doi.org/10.1103/PhysRevC.85.034316.

[8] Disassembling the Nuclear Matrix Elements of the Neutrinoless double beta Decay, J. Menendez et al., Nucl. Phys. A 818, 139 (2009), https://doi.org/10.1016/j.nuclphysa.2008.12.005.

[9] $0 v \beta \beta$ and $2 v \beta \beta$ nuclear matrix elements, quasiparticle random-phase approximation, and isospin symmetry restoration, F. Simkovic et al., Phys. Rev. C 87, 045501 (2013), https://doi.org/10.1103/PhysRevC.87.045501.

[10] $0 v \beta \beta$ and $2 v \beta \beta$ nuclear matrix elements in the interacting boson model with isospin restoration, J. Barea, J. Kotila and F. Iachello, Phys. Rev. C 91, 034304 (2015), https://doi.org/10.1103/PhysRevC.91.034304.

[11] Energy Density Functional Study of Nuclear Matrix Elements for Neutrinoless $\beta \beta$ decay, T. R. Rodriguez and G. Martinez-Pinedo, Phys. Rev. Lett. 105, 252503 (2010), https://doi.org/10.1103/PhysRevLett.105.252503.

[12] Nuclear matrix elements for $0 v \beta \beta$ decays with light or heavy Majorana-neutrino exchange, J. Hyvrinen and J. Suhonen, Phys. Rev. C 91, 024613 (2015), https://doi.org/10.1103/PhysRevC.91.024613.

[13] CUORE: A Cryogenic underground observatory for rare events, C. Arnaboldi et al., [CUORE Collaboration], Nucl. Instrum. Meth. A 518, 775 (2004), https://doi.org/10.1016/j.nima.2003.07.067.

[14] CUORE: A Cryogenic Underground Observatory for Rare Events, R. Ardito et al., [CUORE Collaboration], hep-ex/0501010.

[15] Low-temperature calorimetry for rare decays, E. Fiorini and T. O. Niinikoski, Nuclear Instruments and Methods in Physics Research 224, 83 (1984), https://doi.org/10.1016/0167-5087(84)90449-6.

[16] Physical Principles of Low Temperature Detectors: Ultimate Performance Limits and Current Detector Capabilities, C. Enss, D. McCammon, J. Low Temp Phys 151, 5, (2008), https://doi.org/10.1007/s10909-007-9611-7.

[17] First results on neutrinoless double beta decay of 130Te with the calorimetric CUORICINO experiment, C. Arnaboldi et al., Phys. Lett. B 584, 260 (2004), https://doi.org/10.1016/j.physletb.2004.01.040. 
[18] Results from a search for the $0 v \beta \beta$-decay of 130Te, C. Arnaboldi et al., Phys. Rev. C 78, 035502 (2008), https://doi.org/10.1103/PhysRevC.78.035502.

[19] 130Te neutrinoless double-beta decay with CUORICINO, E. Andreotti et al., Astropart. Phys. 34, 822 (2011), https://doi.org/10.1016/j.astropartphys.2011.02.002.

[20] Production of high purity TeO2 single crystals for the study of neutrinoless double beta decay, C. Arnaboldi et al., J. Cryst. Growth 312, no. 20, 2999 (2010), https://doi.org/10.1016/j.jcrysgro.2010.06.034.

[21] New expectations and uncertainties on neutrinoless double beta decay, S. Dell'Oro, S. Marcocci, F. Vissani, Phys. Rev. D 90, 033005 (2014), https://doi.org/10.1103/PhysRevD.90.033005.

[22] An assembly line for the construction of ultra-radio-pure detectors, E. Buccheri et al., Nucl. Instrum. Meth. A 768, 130 (2014), https://doi.org/10.1016/j.nima.2014.09.046.

[23] Initial performance of the CUORE-0 experiment, D. R. Artusa et al., Eur. Phys. J. C 74, no. 8, 2956 (2014), https://doi.org/10.1140/epjc/s10052-014-2956-6.

[24] Search for Neutrinoless Double-Beta Decay of 130Te with CUORE-0, K. Alfonso et al., Phys. Rev. Lett. 115, 102502 (2015), https://doi.org/10.1103/PhysRevLett.115.102502.

[25] Shell model studies of the 130Te neutrinoless double-beta decay, A. Neacsu and M. Horoi, Phys. Rev. C 91, 024309 (2015), https://doi.org/10.1103/PhysRevC.91.024309.

[26] CUORE Sensitivity to 0v $\beta \beta$ Decay, Alduino, C., Alfonso, K., Artusa, D.R. et al. Eur. Phys. J. C (2017) 77: 532, https://doi.org/10.1140/epjc/s10052-017-5098-9.

[27] Measurement of the two-neutrino double-beta decay half-life of 130Te with the CUORE-0 experiment, Alduino, C., Alfonso, K., Artusa, D.R. et al. Eur. Phys. J. C (2017) 77: 13, https://doi.org/10.1140/epjc/s10052-016-4498-6.

[28] The projected background for the CUORE experiment, Alduino, C., Alfonso, K., Artusa, D.R. et al. Eur. Phys. J. C (2017) 77: 543, https://doi.org/10.1140/epjc/s10052-017-5080-6. 